\title{
Left Atrial Volume Index to Predict Long-Term Recovery of the Left Ventricular Ejection Fraction in Patients with Heart Failure and Reduced Ejection Fraction
} \author{
Tokyo, Japan \\ ORCID: \\ Ayano Yoshida: https://orcid.org/0000-0001-5653-4843 \\ Atsushi Suzuki: https://orcid.org/0000-0002-7566-1749 \\ Tsuyoshi Shiga: https://orcid.org/0000-0003-0358-7196 \\ Sae Tanino: https://orcid.org/0000-0001-8489-7203 \\ Chihiro Saito: https://orcid.org/0000-0003-2250-9337 \\ Kotaro Arai: https://orcid.org/0000-0001-5859-4612 \\ Tsuyoshi Suzuki: https://orcid.org/0000-0001-8920-9326 \\ Kyomi Ashihara: https://orcid.org/0000-0003-4391-7866 \\ Nobuhisa Hagiwara: https://orcid.org/0000-0003-1646-1453
}

Ayano Yoshida', Atsushi Suzuki', Tsuyoshi Shiga ${ }^{1,2}$, Sae Tanino', Chihiro Saito', Kotaro Arai', Tsuyoshi Suzuki', Kyomi Ashihara', Nobuhisa Hagiwara ${ }^{1}$

'Department of Cardiology, Tokyo Women's Medical University, ${ }^{2}$ Department of Clinical Pharmacology and Therapeutics, The Jikei University School of Medicine,

\section{Abstract}

Context: Left ventricular ejection fraction (LVEF) recovery is an important treatment goal for patients with heart failure (HF) and reduced EF (HFrEF); however, the applicability of the left atrial volume index (LAVI) in predicting long-term LVEF recovery in HFrEF remains unknown. Aim: We aimed to assess the predictive value of the LAVI in predicting long-term LVEF recovery in patients hospitalized with HFrEF. Settings and Design: This was a retrospective cohort study. Materials and Methods: We analyzed 70 decompensated patients with HF, hospitalized between 2013 and 2014, with an LVEF $<40 \%$. Patients were categorized into recovered ( $\geq 40 \%$ LVEF and $\geq 10 \%$ improvement in LVEF) and nonrecovered groups, according to an echocardiography-measured LVEF $>3$ years postdischarge. Predictive LAVI values used to predict long-term LVEF improvement were determined. Statistical Analysis: The survival rate was determined using Kaplan-Meier analysis. In receiver operating characteristic curve (ROC) analysis, the area under the curve (AUC) and optimal cutoff values were obtained from several echocardiographic parameters. Univariate and multivariate logistic regression analyses identified predictors of LVEF improvement. Results: Twenty-seven (39\%) patients had recovered LVEFs. During a median follow-up period of 76 (60-80) months, the survival rate was significantly higher in the recovered group (log-rank test, $P=0.001$ ). ROC analysis showed that LAVI's predictive performance in long-term LVEF improvement (AUC $0.78,95 \%$ confidence interval [CI $] 0.66-0.87$ ) was optimal at a cutoff of $35 \mathrm{~g} / \mathrm{m}^{2}$. LAVI $<35 \mathrm{ml} / \mathrm{m}^{2}$ independently predicted LVEF improvement (odds ratio 6.02, 95\% CI 1.26-28.81, $\mathrm{p}=0.025$ ). Conclusions: LAVI is associated with predicting long-term LVEF improvement.

Keywords: Arrhythmia, heart failure, left atrial volume index, left ventricular ejection fraction, major adverse cardiovascular events, prognosis

\section{INTRODUCTION}

Heart failure (HF) is stratified according to the left ventricular ejection fraction (LVEF). ${ }^{[1]}$ Among patients with HF and

Received: 23-06-2021 Revised: 07-08-2021 Accepted: 20-08-2021

Published Online: 25-09-2021

\begin{tabular}{|l|l|}
\hline \multicolumn{3}{c|}{ Access this article online } \\
\hline Quick Response Code: & Website: \\
& \\
http://www.ijcva.com
\end{tabular}

Address for correspondence: Dr. Atsushi Suzuki, Department of Cardiology, Tokyo Women's Medical University, 8-1, Kawada-cho, Shinjuku-ku, Tokyo 162-8666, Japan. E-mail: suzuki.atsushi@twmu.ac.jp

This is an open access journal, and articles are distributed under the terms of the Creative Commons Attribution-NonCommercial-ShareAlike 4.0 License, which allows others to remix, tweak, and build upon the work non-commercially, as long as appropriate credit is given and the new creations are licensed under the identical terms.

For reprints contact: WKHLRPMedknow_reprints@wolterskluwer.com

How to cite this article: Yoshida A, Suzuki A, Shiga T, Tanino S, Saito C, Arai $\mathrm{K}$, et al. Left atrial volume index to predict long-term recovery of the left ventricular ejection fraction in patients with heart failure and reduced ejection fraction. Int J Cardiovasc Acad 2021;7:92-9. 
reduced LVEF (HFrEF), LVEF improvement is important during HF treatment. ${ }^{[2]}$

A larger left atrial diameter (LAD) has been reported to be a predictor of cardiovascular events. ${ }^{[3-6]}$ Left atrium (LA) performance is not unidirectional; therefore, evaluating the size of the LA is preferable in terms of two-dimensionalbased volume. ${ }^{[7]}$ This study aimed to estimate the relationship between LA size, measured using the left atrial volume index (LAVI), and long-term LVEF recovery in discharged patients with HFrEF.

\section{Materials and Methods}

\section{Study design and population}

A Diagnosis Procedure Combination (DPC) database was used in the Heart Institute of Japan Heart Failure study (HIJ-HF II) retrospective, multicenter, cohort study that involved ten participating DPC hospitals in Japan. That study's design and preliminary results have been described elsewhere. ${ }^{[8]}$ That study's protocol was approved by the Institutional Review Boards of Tokyo Women's Medical University. Owing to the HIJ-HF II study's retrospective design, the requirement for written informed consent was waived.

Briefly, 1245 consecutive patients hospitalized for decompensated HF across ten hospitals were enrolled in the study between April 2013 and March 2014. The diagnosis and history of HF were determined by an experienced attending cardiologist and were based on the Framingham study criteria. $^{[9]}$

From the HIJ-HF II cohort, 143 decompensated HF patients with LVEFs $<40 \%$ had been discharged from Tokyo Women's Medical University. Of these, 86 (40\%) patients who had been followed up at Tokyo Women's Medical University and who had undergone an echocardiography examination $>3$ years after discharge were included in our study. Given the challenges in evaluating atrial volume measurements using echocardiography, patients with congenital heart disease, patients with implanted ventricular assist devices, and patients who had previously undergone mitral valve surgery were excluded from this study [Figure 1]. In total, our study comprised 70 patients (50 men; median age, 65 [range, 53-71] years). Echocardiography was performed 54 (range, 48-58) months after discharge to evaluate long-term recovery using LVEF data.

\section{Data collection and endpoints}

Patient background, medical history, blood test results, ultrasound data, and angiographic data were collected by a physician or a trained clinical research coordinator. The primary end point was the LVEF at the long-term follow-up examination after discharge. The secondary end point was all-cause death.

\section{Echocardiography}

We analyzed echocardiographic parameters that had been recorded for patients during their hospitalization. All

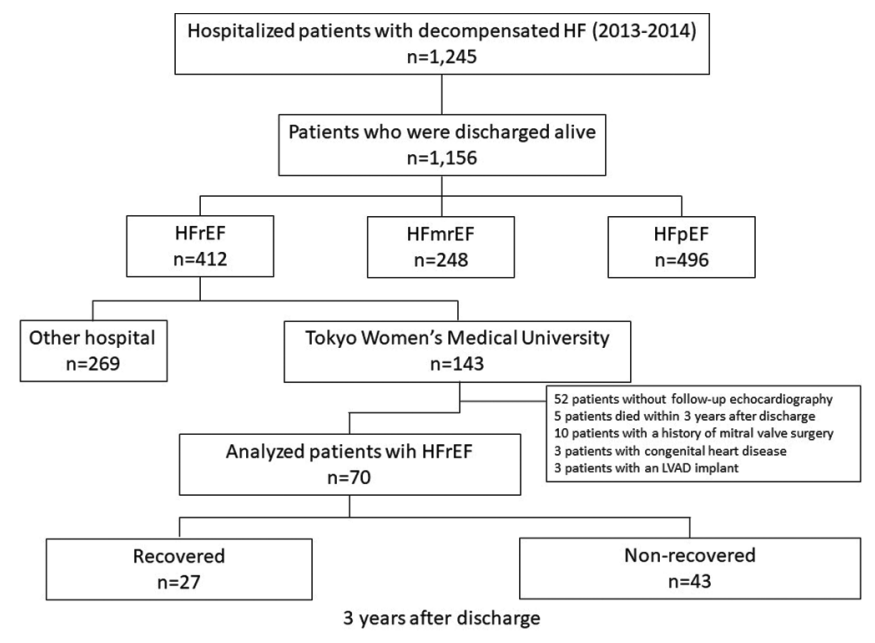

Figure 1: Flow diagram of the study patients. HF: Heart failure; HFmrEF: Heart failure with midrange ejection fraction; HFpEF: Heart failure with preserved ejection fraction; HFrEF: Heart failure with reduced ejection fraction; LVEF: Left ventricular ejection fraction; LVAD Left ventricular-assisted device

images were stored digitally, and the relevant parameters were measured according to the American Society of Echocardiography recommendations. ${ }^{[10]}$ The left ventricular end-diastolic volume (LVEDV), the left ventricular end-systolic volume (LVESV), and the LVEF were calculated by the biplane-disc summation method (modified Simpson's rule), using the apical two- and four-chamber views. The LAD in end-systole was determined using the American Society of Echocardiography standards. ${ }^{[1]]}$ The left atrial volume (LAV) was measured using the bi-apical (two- and four-chamber views) according to the Simpson's rule at the LV end-systole..$^{[12]}$ The LAVI $\left(\mathrm{ml} / \mathrm{m}^{2}\right)$ was defined as the LAV divided by the body surface area $\left(\mathrm{m}^{2}\right)$. The LV mass (LVM) was calculated as the left ventricular diastolic dysfunction (LVDd), interventricular septal thickness (IVST) at end-diastole, and posterior wall thickness (PWT) at end-diastole using the cube formula, ${ }^{[1]}$ as follows:

$\operatorname{LVM}(g)=(0.8 \times 1.04) \times\left([\mathrm{LVDd}+\mathrm{IVST}+\mathrm{PWT}]^{3}-[\mathrm{LVDd}]^{3}\right)$ +0.6 .

The LVM index $\left(\mathrm{g} / \mathrm{m}^{2}\right)$ was defined as the LVM $(\mathrm{g})$ divided by the body surface area $\left(\mathrm{m}^{2}\right)$. The systolic velocities (slo and early diastolic velocities (elo $(\mathrm{cm} / \mathrm{s})$ were measured using tissue Doppler imaging (TDI) on the septal mitral annulus and lateral mitral annulus as a peak velocity in early diastole at the leading edge of the spectral waveform. The E-wave maximum velocity of transmitral flow was measured using pulsed-wave Doppler. Given that $>30 \%$ patients with atrial fibrillation (AF) or ventricular pacing were included in this study, A-wave velocity was excluded. The E/e' ratio was calculated using the E-wave maximum velocity and en of the septal mitral annulus. ${ }^{[13]}$ The deceleration time of the $\mathrm{E}$ velocity was measured as the time interval from the E-wave peak to the velocity decline at baseline. The tricuspid annular plane systolic excursion (TAPSE) was measured in the apical 
four-chamber view through placing the M-mode cursor optimally aligned along the direction of the tricuspid annulus. The peak excursion of the lateral annulus represented the TAPSE ( $\mathrm{mm}$ ). The right ventricular systolic pressure (RVSP) was estimated from the Doppler-derived velocity of the tricuspid regurgitation jet. ${ }^{[12]}$ The color Doppler scale of mitral regurgitation (MR) was evaluated by a clinical ultrasonologist. The MR color jet area was measured on apical four-chamber, apical two-chamber, and long-axis views. To determine the severity of MR, the ratio of the MR color jet region to the LA region (MR/LA ratio) was calculated using both maximum measures. Moderate and severe MR was defined MR/LA ratios of $\geq 0.2$ to $<0.4$ and $\geq 0.4$, respectively. ${ }^{[14]}$

\section{Statistical analysis}

Continuous data are presented as numbers, and categorical data are presented as medians and interquartile ranges. The LVEF was evaluated using echocardiology $>3$ years after discharge and was then used to divide the patients into two groups: the recovered group, defined as an LVEF of $\geq 40 \%$ and $\geq 10 \%$ absolute improvement in LVEF, and the nonrecovered group. Continuous variables were compared using the Wilcoxon rank-sum test, and categorical variables were compared using Fisher's exact test. To evaluate the influence of the LVEF recovery with respect to subsequent death, the survival rate was analyzed using the Kaplan-Meier method. In the receiver operating characteristic curve (ROC) analysis, the area under the curve (AUC) and the optimal cutoff values for several echocardiographic parameters, including LAVI, were determined to predict LVEF improvement after discharge. Univariate and multivariate logistic regression analyses were performed to identify independent predictors of LVEF improvement among echocardiographic parameters. In the multivariate logistic regression analysis, confounding echocardiographic parameters with an AUC of $>0.75$ were used and further adjusted using clinical parameters identified as significant in the univariate analysis. A $P<0.05$ was considered statistically significant. Data analyses were performed with SPSS statistical software (version 22.0, IBM Corp., Armonk, NY, USA).

\section{RESULTS}

\section{Patient characteristics}

Data concerning 70 patients with HFrEF (median age, 64 [range, 53-71] years; males, 71\%; ischemic heart disease, $31 \%$ of patients) were analyzed. The recovered LVEF group comprised $27(39 \%)$ patients. The baseline characteristics at discharge are presented in Table 1. The recovered group had a significantly shorter QRS width at discharge than the nonrecovered group (median: 100 vs. $130 \mathrm{~ms}$, respectively; $P=0.019$ ). Patients in the recovered group were less likely to have ischemic heart disease than those in the nonrecovered group $(P=0.059)$. The proportion of patients who received cardiac implantable electronic devices did not differ between the two groups. Regarding medication at discharge, a greater proportion of patients in the nonrecovered group received diuretics than in the recovered group $(P=0.002)$. There were no between-group differences in terms of the administration rate of beta-blockers, angiotensin-converting enzyme inhibitors, angiotensin II receptor blockers, and mineralocorticoid receptor antagonists.

\section{Prognosis}

During a median follow-up of 76 (60-80) months, 17 (24\%) patients died. The survival rate was significantly higher in patients with recovered LVEFs than in those without [ 5 years, 96 vs. $66 \%, P=0.001$; Figure 2].

\section{Predictors of long-term left ventricular ejection fraction recovery}

The echocardiographic characteristics before discharge are summarized in Table 2. The LV and LA were smaller in size in the recovered group than in the nonrecovered group. Baseline LVEFs were lower in the nonrecovered group. The septal e', and E/e' determined according to the TDI were also lower in the nonrecovered group. In terms of MR rates, the recovered group had a significantly lower rate of MR (moderate or severe) compared to the nonrecovered group.

Results of the univariate logistic regression analysis indicated that several echocardiographic parameters were significantly associated with LVEF recovery [Table 3]. Because some parameters were similar to each other, the following echocardiographic parameters were selected: LVEDV and LVESV as indicators of the LV size, LAVI as an indicator of the LA size, and RVSP as an index of the right ventricular pressure. The nine parameters were significantly associated with the recovered LVEF using a univariate logistic regression method [Table 3]. In the ROC curve analysis, the AUC values for the nine parameters were presented in Table 4. LVEDV, LVES, and LAVI had AUC values of $>0.75$. In the multivariate logistic regression analysis, which we performed using confounding parameters with AUCs $>0.75$ and MR that was found by to be a significant parameter by univariate analysis, $\mathrm{LAVI}<35 \mathrm{ml} / \mathrm{m}^{2}$

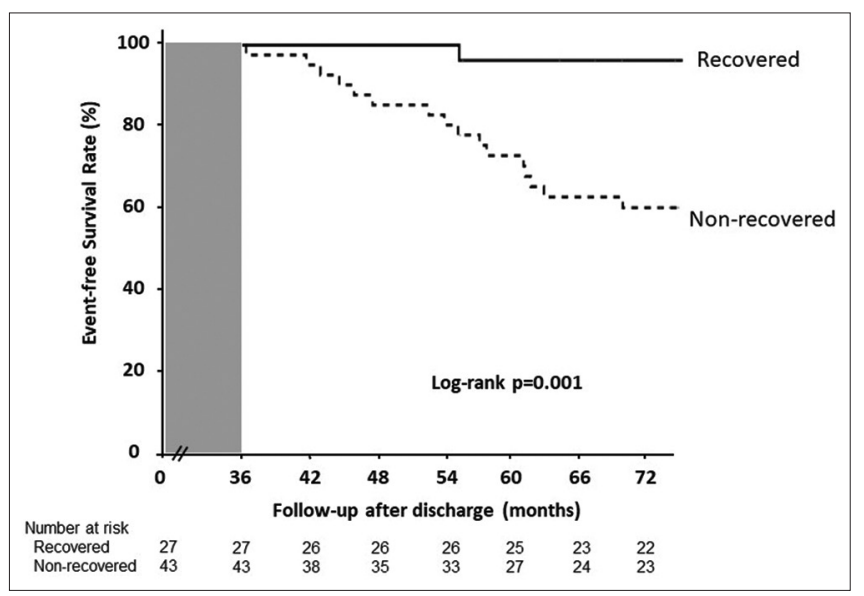

Figure 2: Kaplan-Meier's curves for all-cause death. Survival rates differed significantly between the recovered and nonrecovered left ventricular ejection fraction groups 
Yoshida, et al.: Left atrial volume index and long-term recovery

\begin{tabular}{|c|c|c|c|c|}
\hline Variable & Overall $(n=70)$ & Recovered $(n=27)$ & Nonrecovered $(n=43)$ & $P$ \\
\hline Age (years) & $65(53-71)$ & $63(50-70)$ & $66(56-72)$ & 0.169 \\
\hline Male sex & $50(71)$ & $18(67)$ & $32(74)$ & 0.487 \\
\hline Body mass index $\left(\mathrm{kg} / \mathrm{m}^{2}\right)$ & $24(21-26)$ & $21(20-24)$ & $24(22-26)$ & 0.637 \\
\hline Blood pressure (mmHg) & $108(98-118)$ & $110(106-118)$ & $104(96-118)$ & 0.183 \\
\hline Heart rate (bpm) & $70(60-74)$ & $70(67-77)$ & $68(60-74)$ & 0.168 \\
\hline NYHA Class I/II/III/IV & $5 / 59 / 6 / 0(7 / 84 / 9 / 0)$ & $2 / 25 / 0 / 0(7 / 93 / 0 / 0)$ & $3 / 34 / 6 / 0(7 / 79 / 14 / 0)$ & 0.127 \\
\hline Ischemic heart disease & $22(31)$ & $5(19)$ & $17(40)$ & 0.059 \\
\hline Atrial fibrillation & $21(30)$ & $7(26)$ & $14(33)$ & 0.553 \\
\hline Sustained VT/VF & $4(6)$ & $1(4)$ & $3(7)$ & 0.555 \\
\hline $\mathrm{eGFR}<60 \mathrm{~mL} / \mathrm{min} / 1.73 \mathrm{~m}^{2}$ & $23(33)$ & $6(22)$ & $17(40)$ & 0.111 \\
\hline Plasma BNP (pg/mL) & $243(155-469)$ & $178(48-490)$ & $293(187-525)$ & 0.255 \\
\hline CRT-P/CRT-D & $11(16)$ & $4(15)$ & $7(16)$ & 0.870 \\
\hline ICD & $3(4)$ & $1(4)$ & $2(5)$ & 0.848 \\
\hline Atrial fibrillation & $9(15)$ & $3(13)$ & $6(16)$ & 0.687 \\
\hline QRS complex duration $(\mathrm{m} / \mathrm{s})$ & $125(100-160)$ & $100(90-160)$ & $130(110-160)$ & 0.019 \\
\hline \multicolumn{5}{|l|}{ Medications } \\
\hline ACE inhibitors/ARBs & $60(86)$ & $25(93)$ & $35(81)$ & 0.175 \\
\hline Beta-blockers & $63(90)$ & $26(97)$ & $37(86)$ & 0.138 \\
\hline Diuretics & $51(73)$ & $14(52)$ & $37(86)$ & 0.002 \\
\hline MRAs & $40(57)$ & $14(52)$ & $26(60)$ & 0.479 \\
\hline Digoxin & $17(24)$ & $4(15)$ & $13(30)$ & 0.133 \\
\hline
\end{tabular}

(odds ratio 6.02, 95\% CI 1.26-28.81, $\mathrm{p}=0.025$ ) was identified as an independent predictor of the recovered LVEF [Table 5].

\section{DISCUSSION}

This study aimed to assess the value of transthoracic echocardiography in predicting long-term LVEF recovery among Japanese patients with decompensated HF and HFrEF. Our results, despite several limitations, showed that the LAVI was an independent predictor of long-term LVEF recovery among patients with HFrEF.

\section{Left ventricular ejection fraction recovery}

In this single-center study, 27/70 (39\%) discharged patients with HFrEF had recovered LVEFs after a median follow-up period of 4.5 years. Definitions concerning LVEF improvement, background patient characteristics, number of patients, and follow-up periods have differed among previously reported studies, and the reported number of patients with HF and improved LVEFs has ranged from $10 \%{ }^{[2]}$ to $40 \% .^{[15]}$ The percentage of patients with improved LVEFs (defined as LVEF $<40 \%$ ) in our study was $41 \%$ (29 patients), which was similar to that reported by Teeter et al. ${ }^{[15]}$ After echocardiography at the mid-term follow-up (mean, $24 \pm 7$ months; range, 9-36 months), Merlo et al. reported that a higher proportion (37\%) of patients with nonischemic cardiomyopathy showed LVEF improvement. ${ }^{[16]}$ In our study, the number of patients with nonischemic heart disease was higher in patients in the nonrecovered LVEF group. Patients in the recovered group had a significantly shorter QRS width. Kimura et al. reported that a narrow QRS complex in patients with dilated cardiomyopathy was a predictor of improvement in cardiac function. ${ }^{[17]}$ Although there was an increase in the use of medications capable of antagonizing the neurohormonal system among patients with HFrEF in our HIJ-HF $^{[18]}$ and CHART ${ }^{[19]}$ cohorts, we considered that, based on the HF treatment guidelines, ${ }^{[1,20]}$ the QRS complex on the baseline evaluation electrocardiogram could be associated with myocardial damage in patients with HFrEF.

\section{Prognosis}

Patients in the recovered group were found to have a better long-term prognosis than those in the nonrecovered group. Several studies have reported that LVEF change is an independent predictor of mortality. In a prospective study by Lupón et al., ${ }^{[21]}$ patients with improved LVEFs (defined as LVEF of $\geq 45 \%$ at 1 -year follow-up) had significantly improved mortality and morbidity rates than patients with HF with a preserved ejection fraction and those with HFrEF. In a retrospective cohort study using a $40 \%$ LVEF cutoff point, Kalogeropoulos et al. reported that patients with improved LVEF had a lower mortality rate, less frequent hospitalizations, and fewer composite end points after 3 years. ${ }^{[22]}$ Furthermore, Savarese et al. reported that an increased LVEF was associated with a lower risk of mortality in patients with HFrEF, whereas a decreased LVEF was associated with a higher risk of mortality. ${ }^{[23]}$ However, in these previous reports, $>50 \%$ of patients had ischemic heart disease, whereas only $31 \%$ of 


\begin{tabular}{|c|c|c|c|c|}
\hline Variable & Overall $(n=70)$ & Recovered ( $n=27)$ & Nonrecovered $(n=43)$ & $P$ \\
\hline LVDd (mm) & $62(56-67)$ & $56(52-58)$ & $65(61-70)$ & $<0.001$ \\
\hline LVDs (mm) & $53(47-61)$ & $47(41-49)$ & $57(52-64)$ & $<0.001$ \\
\hline LVEDV (mL) & $195(149-241)$ & $156(124-192)$ & $216(181-278)$ & 0.001 \\
\hline LVESV (mL) & $136(99-180)$ & $103(82-126)$ & $154(133-219)$ & $<0.001$ \\
\hline LVEF (\%) & $30(23-34)$ & $34(29-36)$ & $28(22-31)$ & 0.001 \\
\hline IVST (mm) & $8(7-10)$ & $8(7-10)$ & $8(7-10)$ & 0.389 \\
\hline LVPWT (mm) & $8(8-9)$ & $9(8-10)$ & $8(7-9)$ & 0.398 \\
\hline $\mathrm{LAD}(\mathrm{mm})$ & $44(38-51)$ & $38(35-45)$ & $46(42-53)$ & 0.002 \\
\hline LAV (mm) & $87(61-111)$ & $58(46-104)$ & $95(72-114)$ & 0.004 \\
\hline LAVI $\left(\mathrm{ml} / \mathrm{m}^{2}\right)$ & $50(34-65)$ & $34(30-49)$ & $59(46-69)$ & 0.001 \\
\hline LVMI $\left(\mathrm{g} / \mathrm{m}^{2}\right)$ & $118(97-149)$ & $100(83-120)$ & $130(110-162)$ & 0.001 \\
\hline TAPSE (mm) & $15(12-18)$ & $15(13-19)$ & $15(12-18)$ & 0.739 \\
\hline Septal s' $(\mathrm{cm} / \mathrm{s})$ & $3.8(3.2-4.7)$ & $4.2(3.5-4.8)$ & $3.8(3.1-4.7)$ & 0.319 \\
\hline Septal e' $(\mathrm{cm} / \mathrm{s})$ & $4.5(3.2-5.4)$ & $5.1(4.0-6.4)$ & $3.7(3.0-4.9)$ & 0.002 \\
\hline Lateral s' $(\mathrm{cm} / \mathrm{s})$ & $4.6(3.9-5.4)$ & $5.3(4.0-6.1)$ & $4.3(3.8-5.0)$ & 0.176 \\
\hline Lateral e' $(\mathrm{cm} / \mathrm{s})$ & $5.8(3.7-8.4)$ & $6.7(4.9-11.1)$ & $5.4(3.6-7.5)$ & 0.067 \\
\hline E wave $(\mathrm{cm} / \mathrm{s})$ & $70(55-95)$ & $66(55-92)$ & $76(55-99)$ & 0.794 \\
\hline $\mathrm{E} / \mathrm{e}^{\prime}$ & $15(13-21)$ & $13(11-16)$ & $18(15-27)$ & 0.017 \\
\hline DT (msec) & $158(124-208)$ & $160(129-264)$ & $156(123-191)$ & 0.066 \\
\hline $\mathrm{TRV} \max (\mathrm{m} / \mathrm{s})$ & $2.5(2.3-2.9)$ & $2.3(2.1-2.7)$ & $2.6(2.4-3.0)$ & 0.012 \\
\hline RVSP (mmHg) & $34(30-44)$ & $31(28-40)$ & $39(33-52)$ & 0.005 \\
\hline MR (moderate or severe) & $19(27)$ & $3(11)$ & $16(37)$ & 0.015 \\
\hline \multicolumn{5}{|c|}{$\begin{array}{l}\text { Values are } n(\%) \text { or median (IQR). IQR: Interquartile range, AR: Aortic valve regurgitation, AS: Aortic valve stenosis, DT: Deceleration time of early } \\
\text { diastolic inflow, e': Peak early diastolic annular velocity, E/A: Ratio of peak transmitral early diastolic filling velocity to peak transmitral atrial filling } \\
\text { velocity, E/e': Ratio of peak transmitral early diastolic filling velocity to peak early diastolic mitral annular velocity, IVST: Interventricular septum } \\
\text { thickness, LAD: Left atrial dimension, LAV: Left atrial volume, LAVI: Left atrial volume index, LVDd: Left ventricular end-diastolic dimension, } \\
\text { LVDs: Left ventricular end-systolic dimension, LVEDV: Left ventricular end-diastolic volume, LVEDVI: Left ventricular end-diastolic volume index, } \\
\text { LVEF: Left ventricular ejection fraction, LVESV: Left ventricular end-systolic volume, LVESVI: Left ventricular end-systolic volume index, LVMI: Left } \\
\text { ventricular mass index, LVPWT: Left ventricular posterior wall thickness, MR: Mitral regurgitation, N/A: Not available, RV: Right ventricular, } \\
\text { RVSP: Right ventricular systolic pressure, s': Lowest, TAPSE: Tricuspid annular plane systolic excursion, TRV: Tricuspid regurgitant velocity }\end{array}$} \\
\hline
\end{tabular}

patients had ischemic heart disease in our study. Zecchin et al. reported that approximately $66 \%$ of patients with new-onset idiopathic dilated cardiomyopathy showed LVEF improvement 3-9 months later following optimal medical therapy, with excellent long-term prognoses during the $110 \pm 63$-month follow-up period. ${ }^{[24]}$ Our study findings showed that long-term change in the LVEF was also associated with mortality. We defined LVEF recovery as a follow-up LVEF of $\geq 40 \%$ and $\geq 10 \%$ absolute improvement in the LVEF. These indicators in relation to the LVEF have been reported to correlate with a decrease in LV volumes. ${ }^{[25]}$ It is necessary for patients with HF to undergo regular follow-ups and to have optimal treatment confirmed, including drug titration or device therapy, depending on the LVEF assessed during long-term follow-up.

\section{Left atrial volume index and left ventricular ejection fraction recovery}

In patients with congestive HF (CHF), an increased LAV typically reflects high LV filling pressure. ${ }^{[26]}$ During diastole, the LA is exposed to pressure from the LV. Increased LV stiffness or noncompliance increases the LA pressure to maintain proper LV filling, and increased atrial wall tension results in atrial dilation and atrial myocardial extension. ${ }^{[27]}$ Consequently, the LAV increases with the severity of diastolic dysfunction. ${ }^{[28,29]}$ Sustained LV diastolic dysfunction causes stretching of cardiac myocytes, leading to LV remodeling and further neurohormonal stimulation. Therefore, the LAV is a simple noninvasive assessment of LV diastolic function. ${ }^{[7,26]}$ Several studies have found that LA dilation was predictive of cardiovascular outcomes, such as AF, CHF, cardiovascular death, and stroke. ${ }^{[4-6]}$ Rossi et al. reported that smaller baseline LAVs before cardiac resynchronization therapy (CRT) were significantly associated with LV reverse remodeling after CRT. ${ }^{[30]}$ When evaluating the LA, the volumetric measurement of the LA should be preferred over the LA diameter to avoid underestimation ${ }^{[31]}$ However, the predictive value of the LAVI has not been fully evaluated in patients with HFrEF. Our study showed that the LAVI was an independent predictor of improved LVEF after discharge. Regarding the parameters related to the LV end-diastolic pressure before discharge, the E wave, E/A, and E/e' in patients with LAVIs of $<35 \mathrm{ml} / \mathrm{m}^{2}$ were significantly lower than those in patients with LAVIs of $\geq 35 \mathrm{ml} / \mathrm{m}^{2}$ (57 [37-69] vs. 82 [62-103] cm/s; 0.9 [0.7-1.3] vs. 1.7 [1.2-3.0]; and 12.8 [10.3-15.5] vs. 16.2 [13.6-24.1], respectively). In addition, DT was significantly higher in patients with larger LAVIs than in those with smaller LAVIs (208 [143-274] vs. 150 [120-183] ms, respectively). Further, LA enlargement may be associated with increased LV 
end-diastolic pressure and LV diastolic dysfunction in patients with HFrEF before discharge. LA enlargement and dysfunction are common in patients with $\mathrm{AF} .{ }^{[32,33]}$ In our study, although $30 \%$ of patients had AF, the LAVI was also associated with LVEF improvement after adjustment for the prevalence of AF. MR is another related factor influencing volume overload, thus leading to LA enlargement. ${ }^{[34]}$ It has also been reported that the degree of MR is associated with the LAV independent of the presence or absence of AF. A greater number of patients in the recovered group had MR; however, LAVI was independently

\begin{tabular}{|c|c|c|c|}
\hline & OR & $95 \% \mathrm{Cl}$ & $P$ \\
\hline LVEDV (1 ml decrease) & 1.02 & $1.01-1.04$ & 0.001 \\
\hline LVESV (1 ml decrease) & 1.01 & $1.01-1.02$ & 0.003 \\
\hline LVEF (1\% decrease) & 1.14 & $1.04-1.25$ & 0.004 \\
\hline IVST (1 mm increase) & 1.19 & $0.93-1.54$ & 0.170 \\
\hline LVPWT (1 mm increase) & 1.25 & $0.90-1.76$ & 0.185 \\
\hline LAVI ( $1 \mathrm{ml} / \mathrm{m}^{2}$ decrease $)$ & 1.05 & $1.02-1.08$ & 0.002 \\
\hline LVMI ( $1 \mathrm{~g} / \mathrm{m}^{2}$ increase $)$ & 0.98 & $0.96-0.99$ & 0.006 \\
\hline TAPSE (1 mm decrease) & 0.97 & $0.78-1.21$ & 0.797 \\
\hline Septal s' $(1 \mathrm{~cm} / \mathrm{s}$ decrease $)$ & 1.26 & $0.77-2.06$ & 0.348 \\
\hline Septal e' $(1 \mathrm{~cm} / \mathrm{s}$ decrease $)$ & 1.51 & $1.09-2.10$ & 0.012 \\
\hline Lateral s' $(1 \mathrm{~cm} / \mathrm{s}$ decrease $)$ & 1.25 & $0.85-1.86$ & 0.257 \\
\hline Lateral e' $(1 \mathrm{~cm} / \mathrm{s}$ decrease $)$ & 1.14 & $0.97-1.34$ & 0.106 \\
\hline E wave $(1 \mathrm{~cm} / \mathrm{s}$ decrease $)$ & 0.99 & $0.97-1.01$ & 0.375 \\
\hline E/e' (1 unit decrease) & 0.91 & $0.85-0.98$ & 0.015 \\
\hline DT (1 ms increase) & 1.01 & $1.00-1.02$ & 0.035 \\
\hline RVSP (1 mmHg increase) & 0.92 & $0.86-0.97$ & 0.005 \\
\hline MR (moderate or severe) & 0.21 & $0.06-0.81$ & 0.024 \\
\hline
\end{tabular}

CI: Confidence interval, DT: Deceleration time of early diastolic inflow, e': Peak early diastolic annular velocity, E/e': Ratio of peak transmitral early diastolic filling velocity to peak early diastolic mitral annular velocity, IVST: Interventricular septum thickness, LAVI: Left atrial volume index, LVEDV: Left ventricular end-diastolic volume, LVEF: Left ventricular ejection fraction, LVESV: Left ventricular end-systolic volume, LVMI: Left ventricular mass index, LVPWT: Left ventricular posterior wall thickness, MR: Mitral regurgitation, OR: Odds ratio, RVSP: Right ventricular systolic pressure, s': Lowest, TAPSE: Tricuspid annular plane systolic excursion associated with LVEF improvement by multivariate analysis. ${ }^{[35]}$ Although baseline LV size and LV function were found to be important factors, as shown in the univariate logistic regression analysis (LVEDV, LVESV, and LVEF), the definition of LVEF improvement was different from that in previous studies, ${ }^{[21-23]}$ the number of patients with nonischemic heart disease was high, and the sample size of this study was small. Therefore, we recommend that LAVI should be investigated during the echocardiographic evaluation of hospitalized patients with HF.

\section{Study limitations}

This study had several limitations. First, this was a single-center, retrospective study. We could not determine the influence of treatment, including HF medications and devices at the time of echocardiography. Although echocardiography is performed routinely in our institution, selection bias may have affected the results owing to variations in the date of the follow-up echocardiography after discharge. Second, the number of study patients was very small. Because limited data were available, we excluded patients who visited other institutions after discharge. Further research is necessary to determine the mechanisms involved in the association between LVEF improvement and LAVI, and a prospective study is needed to test the prognostic importance of the LAVI in patients with HFrEF.

\section{Conclusions}

Our study findings indicated that the baseline LAVI was independently associated with LVEF recovery during long-term follow-up in patients with HFrEF. The size of the LA can be considered not only in terms of diastolic function but also as a predictor of long-term LVEF recovery among patients with HFrEF.

\section{Acknowledgments}

We thank Ms. Emi Sawada for her assistance and project management.

\section{Financial support and sponsorship}

This work was supported by JSPS KAKENHI Grant Number $20 \mathrm{~K} 08499$.

Table 4: Area under the curve for relevant echocardiographic variables for left ventricular ejection fraction recovery

\begin{tabular}{lcccccc}
\hline & AUC & Cut-off & Sensitivity (\%) & Specificity (\%) & Positive likelihood ratio & Negative likelihood ratio \\
\hline LVEDV $(\mathrm{mL})$ & $0.78(0.67-0.87)$ & $\leq 201$ & $89(71-98)$ & $63(47-77)$ & $2.4(1.6-53.6)$ & $0.2(0.1-0.5)$ \\
LVESV $(\mathrm{mL})$ & $0.82(0.70-0.90)$ & $\leq 126$ & $78(58-91)$ & $81(67-92)$ & $4.2(2.2-8.1)$ & $0.3(0.1-0.6)$ \\
LVEF $(\%)$ & $0.74(0.62-0.84)$ & $>32$ & $66(46-82)$ & $83(68-93)$ & $3.8(1.9-7.9)$ & $0.4(0.2-0.7)$ \\
LAVI $\left(\mathrm{ml} / \mathrm{m}^{2}\right)$ & $0.78(0.66-0.87)$ & $<35$ & $60(41-77)$ & $93(81-99)$ & $8.6(2.8-26.6)$ & $0.4(0.3-0.7)$ \\
LVMI $\left(\mathrm{g} / \mathrm{m}^{2}\right)$ & $0.70(0.58-0.81)$ & $\leq 107$ & $55(36-74)$ & $78(62-89)$ & $2.5(1.3-4.9)$ & $0.6(0.4-0.9)$ \\
Septal e' $(\mathrm{cm} / \mathrm{s})$ & $0.68(0.55-0.78)$ & $>5.2$ & $44(26-64)$ & $88(74-96)$ & $3.7(1.5-9.2)$ & $0.6(0.4-0.9)$ \\
E/e & $0.73(0.61-0.83)$ & $\leq 14.6$ & $69(49-85)$ & $76(60-88)$ & $2.8(1.6-5.1)$ & $0.4(0.2-0.7)$ \\
DT $(\mathrm{m} / \mathrm{s})$ & $0.62(0.49-0.73)$ & $>215$ & $38(21-58)$ & $93(80-99)$ & $5.2(1.6-17.0)$ & $0.7(0.5-0.9)$ \\
RVSP $(\mathrm{mmHg})$ & $0.72(0.59-0.82)$ & $\leq 31.2$ & $58(37-77)$ & $79(64-91)$ & $2.8(1.4-5.7)$ & $0.5(0.3-0.9)$ \\
\hline AVC: $\mathrm{Ar}$
\end{tabular}

AUC: Area under the curve, DT: Deceleration time of early diastolic inflow, e': Peak early diastolic annular velocity, E/e': Ratio of peak transmitral early diastolic filling velocity to peak early diastolic mitral annular velocity, LAVI: Left atrial volume index, LVEDV: Left ventricular end-diastolic volume, LVEF: Left ventricular ejection fraction, LVESV: Left ventricular end-systolic volume, LVMI: Left ventricular mass index, RVSP: Right ventricular systolic pressure 


\begin{tabular}{|c|c|c|c|}
\hline & OR & $95 \% \mathrm{Cl}$ & $P$ \\
\hline $\operatorname{LVEDV~}(\leq 201 \mathrm{ml})$ & 4.26 & $0.63-28.78$ & 0.137 \\
\hline LVESV $(\leq 126 \mathrm{ml})$ & 3.33 & $0.60-18.41$ & 0.167 \\
\hline LAVI $\left(<35 \mathrm{ml} / \mathrm{m}^{2}\right)$ & 6.02 & $1.26-28.81$ & 0.025 \\
\hline MR (moderate or severe) & 0.53 & $0.10-2.73$ & 0.450 \\
\hline
\end{tabular}

LAVI: Left atrial volume index, LVEDVI: Left ventricular end-diastolic volume index, LVESVI: Left ventricular end-systolic volume index, MR: Mitral regurgitation, OR: Odds ratio, CI: Confidence interval

\section{Conflicts of interest}

There are no conflicts of interest.

\section{References}

1. Ponikowski P, Voors AA, Anker SD, Bueno H, Cleland JG, Coats AJ, et al. 2016 ESC Guidelines for the diagnosis and treatment of acute and chronic heart failure: The Task Force for the diagnosis and treatment of acute and chronic heart failure of the European Society of Cardiology (ESC) Developed with the special contribution of the Heart Failure Association (HFA) of the ESC. Eur Heart J 2016;37:2129-200.

2. Basuray A, French B, Ky B, Vorovich E, Olt C, Sweitzer NK, et al. Heart failure with recovered ejection fraction: Clinical description, biomarkers, and outcomes. Circulation 2014;129:2380-7.

3. Kizer JR, Bella JN, Palmieri V, Liu JE, Best LG, Lee ET, et al. Left atrial diameter as an independent predictor of first clinical cardiovascular events in middle-aged and elderly adults: The Strong Heart Study (SHS). Am Heart J 2006;151:412-8.

4. Benjamin EJ, D'Agostino RB, Belanger AJ, Wolf PA, Levy D. Left atrial size and the risk of stroke and death. The Framingham Heart Study. Circulation 1995;92:835-41.

5. Sabharwal N, Cemin R, Rajan K, Hickman M, Lahiri A, Senior R. Usefulness of left atrial volume as a predictor of mortality in patients with ischemic cardiomyopathy. Am J Cardiol 2004;94:760-3.

6. Lim TK, Dwivedi G, Hayat S, Majumdar S, Senior R. Independent value of left atrial volume index for the prediction of mortality in patients with suspected heart failure referred from the community. Heart 2009;95:1172-8.

7. Tsang TS, Abhayaratna WP, Barnes ME, Miyasaka Y, Gersh BJ, Bailey KR, et al. Prediction of cardiovascular outcomes with left atrial size: Is volume superior to area or diameter? J Am Coll Cardiol 2006;47:1018-23.

8. Shiga T, Suzuki A, Haruta S, Mori F, Ota Y, Yagi M, et al. Clinical characteristics of hospitalized heart failure patients with preserved, mid-range, and reduced ejection fractions in Japan. ESC Heart Fail 2019;6:475-86.

9. Ho KK, Anderson KM, Kannel WB, Grossman W, Levy D. Survival after the onset of congestive heart failure in Framingham Heart Study subjects. Circulation 1993;88:107-15.

10. Mitchell C, Rahko PS, Blauwet LA, Canaday B, Finstuen JA, Foster MC, et al. Guidelines for performing a comprehensive transthoracic echocardiographic examination in adults: Recommendations from the American Society of Echocardiography. J Am Soc Echocardiogr 2019;32:1-64.

11. Lang RM, Badano LP, Mor-Avi V, Afilalo J, Armstrong A, Ernande L, et al. Recommendations for cardiac chamber quantification by echocardiography in adults: An update from the American Society of Echocardiography and the European Association of Cardiovascular Imaging. J Am Soc Echocardiogr 2015;28:1-39.e14.

12. Rudski LG, Lai WW, Afilalo J, Hua L, Handschumacher MD, Chandrasekaran $\mathrm{K}$, et al. Guidelines for the echocardiographic assessment of the right heart in adults: A report from the American Society of Echocardiography endorsed by the European Association of Echocardiography, a registered branch of the European Society of Cardiology, and the Canadian Society of Echocardiography. J Am Soc Echocardiogr 2010;23:685-713.

13. Nagueh SF, Smiseth OA, Appleton CP, Byrd BF $3^{\text {rd }}$, Dokainish H, Edvarsen $\mathrm{T}$, et al. Recommendations for the evaluation of left ventricular diastolic function by echocardiography: An update from the American Society of Echocardiography and the European Association of Cardiovascular Imaging. J Am Soc Echocardiogr 2016;29:277-314.

14. Zoghbi WA, Enriquez-Sarano M, Foster E, Grayburn PA, Kraft CD, Levine RA, et al. Recommendations for evaluation of the severity of native valvular regurgitation with two-dimensional and Doppler echocardiography. J Am Soc Echocardiogr 2003;16:777-802.

15. Teeter WA, Thibodeau JT, Rao K, Brickner ME, Toto KH, Nelson LL, et al. The natural history of new-onset heart failure with a severely depressed left ventricular ejection fraction: Implications for timing of implantable cardioverter-defibrillator implantation. Am Heart J 2012;164:358-64.

16. Merlo M, Pyxaras SA, Pinamonti B, Barbati G, Di Lenarda A, Sinagra G. Prevalence and prognostic significance of left ventricular reverse remodeling in dilated cardiomyopathy receiving tailored medical treatment. J Am Coll Cardiol 2011;57:1468-76.

17. Kimura Y, Okumura T, Morimoto R, Kazama S, Shibata N, Oishi H, et al. A clinical score for predicting left ventricular reverse remodelling in patients with dilated cardiomyopathy. ESC Heart Fail 2021;8:1359-68.

18. Suzuki A, Shiga T, Kawashiro N, Hagiwara N; HIJ-HF II Investigators. Changes in characteristics and outcomes in Japanese patients with heart failure from the 2000s to the 2010s: The HIJ-HF cohorts. J Cardiol 2020;76:132-8.

19. Ushigome R, Sakata Y, Nochioka K, Miyata S, Miura M, Tadaki S, et al. Temporal trends in clinical characteristics, management and prognosis of patients with symptomatic heart failure in Japan - Report from the CHART Studies. Circ J 2015;79:2396-407.

20. Tsutsui $\mathrm{H}$, Isobe M, Ito H, Ito H, Okumura K, Ono M, et al. JCS 2017/ JHFS 2017 guideline on diagnosis and treatment of acute and chronic heart failure - Digest version. Circ J 2019;83:2084-184.

21. Lupón J, Díez-López C, de Antonio M, Domingo M, Zamora E, Moliner P, et al. Recovered heart failure with reduced ejection fraction and outcomes: A prospective study. Eur J Heart Fail 2017;19:1615-23.

22. Kalogeropoulos AP, Fonarow GC, Georgiopoulou V, Burkman G, Siwamogsatham S, Patel A, et al. Characteristics and outcomes of adult outpatients with heart failure and improved or recovered ejection fraction. JAMA Cardiol 2016;1:510-8.

23. Savarese G, Vedin O, D’Amario D, Uijl A, Dahlström U, Rosano G, et al. Prevalence and prognostic implications of longitudinal ejection fraction change in heart failure. JACC Heart Fail 2019;7:306-17.

24. Zecchin M, Merlo M, Pivetta A, Barbati G, Lutman C, Gregori D, et al. How can optimization of medical treatment avoid unnecessary implantable cardioverter-defibrillator implantations in patients with idiopathic dilated cardiomyopathy presenting with "SCD-HeFT criteria?". Am J Cardiol 2012;109:729-35.

25. Wilcox JE, Fang JC, Margulies KB, Mann DL. Heart failure with recovered left ventricular ejection fraction: JACC scientific expert panel. J Am Coll Cardiol 2020;76:719-34.

26. Simek CL, Feldman MD, Haber HL, Wu CC, Jayaweera AR, Kaul S. Relationship between left ventricular wall thickness and left atrial size: Comparison with other measures of diastolic function. J Am Soc Echocardiogr 1995;8:37-47.

27. Greenberg B, Chatterjee K, Parmley WW, Werner JA, Holly AN. The influence of left ventricular filling pressure on atrial contribution to cardiac output. Am Heart J 1979;98:742-51.

28. Tsang TS, Barnes ME, Gersh BJ, Bailey KR, Seward JB. Left atrial volume as a morphophysiologic expression of left ventricular diastolic dysfunction and relation to cardiovascular risk burden. Am J Cardiol 2002;90:1284-9.

29. Prichett AM, Mohoney DW, Jacobsen SJ, Rodeheffer RJ, Karon BL, Redfield MM. Diastolic dysfunction and left atrial volume: A population-based study. J Am Coll Cardiol 2005;45:87-92.

30. Rossi L, Malagoli A, Piepoli M, Franchi F, Malavasi V, Casali E, et al. Indexed maximal left atrial volume predicts response to cardiac resynchronization therapy. Int J Cardiol 2013;168:3629-33. 
31. Fu M, Zhou D, Tang S, Zhou Y, Feng Y, Geng Q. Left atrial volume index is superior to left atrial diameter index in relation to coronary heart disease in hypertension patients with preserved left ventricular ejection fraction. Clin Exp Hypertens 2020;42:1-7.

32. Mochizuki A, Yuda S, Oi Y, Kawamukai M, Nishida J, Kouzu H, et al. Assessment of left atrial deformation and synchrony by three-dimensional speckle-tracking echocardiography: Comparative studies in healthy subjects and patients with atrial fibrillation. J Am Soc Echocardiogr 2013;26:165-74.

33. Inaba Y, Yuda S, Kobayashi N, Hashimoto A, Uno K, Nakata T, et al.
Strain rate imaging for noninvasive functional quantification of the left atrium: Comparative studies in controls and patients with atrial fibrillation. J Am Soc Echocardiogr 2005;18:729-36.

34. Enriquez-Sarano M, Akins CW, Vahanian A. Mitral regurgitation. Lancet 2009;373:1382-94.

35. Messika-Zeitoun D, Bellamy M, Avierinos JF, Breen J, Eusemann C, RossiA, et al. Left atrial remodelling in mitral regurgitation-Methodologic approach, physiological determinants, and outcome implications: A prospective quantitative Doppler-echocardiographic and electron beam-computed tomographic study. Eur Heart J 2007;28:1773-81. 